\title{
DACRIOCISTOPLASTIA CON BALÓN CATÉTER EN NIÑOS CON OBSTRUCCIÓN NASOLAGRIMAL CONGÉNITA EN LOS QUE HA FRACASADO EL SONDAJE
}

\section{BALLON DACRYOCYSTOPLASTY (DCP) FOR TREATMENT OF PEDIATRIC PATIENTS WITH CONGENITAL NASOLACRIMAL DUCT OBSTRUCTION AFTER FAILED PRIMARY PROBING}

\author{
ALAÑÓN FJ ${ }^{1}$, ALAÑÓN MA², MARTÍNEZ A², CALERO B ${ }^{3}$, CÁRDENAS M ${ }^{1}$, CALZADO J ${ }^{2}$, \\ SILES MJ ${ }^{2}$, PIMENTEL $\mathrm{E}^{2}$
}

\section{RESUMEN}

Objetivo: Analizar el resultado de la dacriocistoplastia con balón catéter (DCP) en niños en los que han fallado dos sondajes previos.

Material y métodos: Entre octubre de 2004 y junio de 2006 se practica DCP en un estudio prospectivo en 30 pacientes con una edad entre 18 y 72 meses (media 32,4 meses) con obstrucción nasolagrimal congénita en los que ha fallado el sondaje en dos ocasiones.

Se estudia el tipo de obstrucción y se diferencia entre estenosis lagrimal, obstrucción proximal y membranosa.

Se dividen dos grupos según la edad menores y mayores de 36 meses.

Se incluye cuestionario con test de Munk y examen oftalmológico incluyendo el test de desaparición de colorante a los 5 minutos efectuados en los controles a las 2 semanas, 3 meses y 6 meses en todos los casos. Se define éxito cuando se normalizaron todos los síntomas y signos (Munk 0) y en el test de desaparición de colorante hay ausencia de tinción residual (Grado 0).

\section{ABSTRACT}

Purpose: To determine the efficiency of lacrimal balloon catheter dilatation to treat congenital nasolacrimal duct obstruction in children who had failed two lacrimal system probings.

Methods: Between October 2004 and June 2006 we performed a prospective study of balloon catheter dilatation for congenital nasolacrimal duct obstruction in 30 lacrimal systems.

The mean age was 32.4 months (range 18-72 months). The patients were divided into 3 types of obstructions: partial, proximal and simple membranous at the valve of Hasner and into 2 age categories: category 1 (18-36 months) and category 2 (> 36 months). The patients were evaluated 2 weeks, 3 months and 6 months after balloon catheter dilatation, with Munk's score and ophthalmic evaluation using a dye disappearance test being assessed.

Clinical patency was defined as a complete resolution of signs and symp-toms (Munk 0) and a negative disappearance test (grade 0 ).

We used the chi-squared test with Yates' correction for statistical analysis.

\footnotetext{
Recibido: 21/7/06. Aceptado: 19/6/07.

Instituto Lagrimal Internacional. Jaén. España.

1 Licenciado en Medicina.

2 Doctor en Medicina.

3 Licenciado en Matemáticas.

Correspondencia:

Félix J. Alañón Fernández

C/. Plaza de los Jardinillos, 8, 1. ${ }^{\circ} \mathrm{D}$

23004 Jaén

España

E-mail: sjaf@supercable.es
} 
Se utilizó el test de la chi-cuadrado con corrección de Yates para el análisis estadístico.

Resultados: En el 83,33\% de los pacientes se obtuvo buenos resultados.

El tipo de obstrucción no se relacionó con diferentes resultados. La edad del paciente tampoco afectó a la bondad del resultado (test de la chi-cuadrado a un nivel de confianza del 95\%).

No hubo efectos secundarios ni complicaciones.

Conclusión: La DCP es una técnica quirúrgica segura, sencilla y efectiva para el tratamiento de la obstrucción lagrimal congénita con fracasos de dos sondajes previos.

Palabras clave: Dacriocistoplastia, balón catéter, dilatación, obstrucción nasolagrimal congénita, sondaje fallido.
Results: The procedure was successful in $83.33 \%$ of the cases and there were no complications.

Statistical analysis showed no significant difference between the 3 types of obstructions treated, and age at treatment did not affect the outcome.

Conclusion: DCP is a safe, effective therapy, easy and fast to perform and should be used in the treatment of congenital nasolacrimal duct obstruction after failure of two lacrimal system probings (Arch Soc Esp Oftalmol 2007; 82: 609-000).

Key words: Dacryocystoplasty, balloon catheter, dilatation, failed prob-ing, congenital nasolacrimal duct obstruction.

\section{INTRODUCCIÓN}

La obstrucción lagrimal congénita constituye el motivo más frecuente de consulta al oftalmólogo en menores de 1 año (1).

La dacriocistoplastia (DCP) con balón se inspira en las angioplastias transluminares. El catéter fue descrito en 1964 por Dotter y Judkins y utilizado por primera vez por Grüntzing y Hooff en 1974 para estenosis arteriales de origen ateromatoso. Para patología de la vía lagrimal en un principio, se aplicó un balón de angioplastia en adultos con epífora bajo control radiológico. Becker tras su utilización en adultos diseñó un catéter para niños publicando sus resultados en 1991 (2).

El objetivo de nuestro trabajo es evaluar la DCP en pacientes en los que han fallado dos sondajes estudiando el tipo de obstrucción y la edad del paciente.

\section{SUJETOS, MATERIAL Y MÉTODOS}

Entre octubre de 2004 y junio de 2006 se realizó un estudio prospectivo con 30 pacientes con fracaso de dos sondajes previos y edades comprendidas entre 18 y 72 meses.

El diagnóstico de fracaso se basó en la persistencia de lagrimeo, secreción mucosa o purulenta, reflujo de secreción a la presión digital en región cantal interna, costras, aglutinación de las pestañas y ausencia de desaparición del colorante tras instilar una gota de fluoresceína al $2 \%$ en fondo de saco conjuntival a los 5 minutos. La epífora se cuantificó según la escala de Munk en el pre y postoperatorio de todos los pacientes (tabla I).

No fueron incluidos en el estudio pacientes con dacriocistocele, dacriocistitis aguda, fístulas dacriocutáneas, traumatismos en esta región, alteraciones de puntos y canalículos o alteraciones craneofaciales.

Se clasificaron a los pacientes en dos grupos según la edad: grupo 1 de 18 a 36 meses y grupo 2 mayores de 36 meses.

Después de obtener el consentimiento informado verbal y escrito por parte del tutor se practicó DCP bajo anestesia general por los mismos cirujanos (AF, AM). Se colocó una torunda de algodón con Tetracaína al $1 \%$ y Epinefrina al 1/100.000 en el meato inferior 5 minutos antes de la cirugía. Tras dilatar el punto lagrimal superior se lavó el sistema lagrimal con suero fisiológico hasta que no queden restos de

\section{Tabla I. Escala de Munk}

Grado 0 Ausencia de epífora

Grado 1 Epífora ocasional con necesidad de secado menor de 2 veces al día

Grado 2 Epífora con necesidad de secado entre 2 y 4 veces al día

Grado 3 Epífora con necesidad de secado entre 5-10 veces al día

Grado 4 Epífora con necesidad de secado mayor de 10 veces al día 
secreción mucosa o purulenta, seguidamente se introducía una sustancia viscoelástica diferenciando si es estenosis u obstrucción nasolagrimal según pasara a meato inferior o no el viscoelástico.

Introduciendo una sonda de Bowman del 0 por punto y canalículo superior se percibe el nivel y tipo de obstrucción.

Se define obstrucción membranosa si al pasar la sonda se produce una sensación de chasquido con libre pasaje posterior. Una obstrucción más extensa y proximal a la válvula de Hasner produce una firme sensación de obstrucción, sensación de hueso o sensación de paso de la sonda a través de papel de lija, pasos repetidos no alteran esta sensación.

Se insertó de forma anterógrada un Lacricath Balloon Catheter (Quest Medical, Inc. Atrion Company, Allen, Texas) a través de punto lagrimal y canalículo superior pasándolo a través de saco y conducto nasolagrimal comprobando su correcta localización mediante visualización directa con un endoscopio de $2,7 \mathrm{~mm}$ y $30^{\circ}$ sin necesidad de fluoroscopia. Coincidiendo punto lagrimal superior con la marca proximal del catéter (fig. 1).

Un balón catéter flexible de 2 milímetros de diámetro $(0,60 \mathrm{~mm}$. desinflado $)$ y 13 milímetros de largo fue usado en pacientes de 30 meses o menos. Un balón catéter de 3 milímetros de diámetro $(0,65 \mathrm{~mm}$ desinflado) y 15 milímetros de largo fue insertado en pacientes mayores de 30 meses (fig. 2). Una bomba de presión hidrostática con manómetro para su monitorización, similar a las de angioplastia, fue conectada al balón catéter (Quest Medical, Inc. Atrion Company, Allen, Texas).

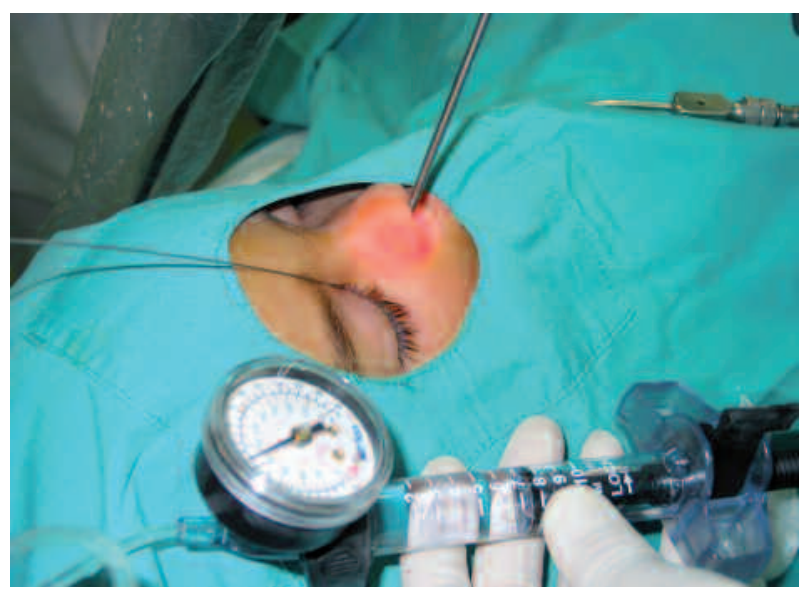

Fig. 1: Introducción anterógrada del balón. Conexión al manómetro. Control endoscópico de su correcta ubicación.

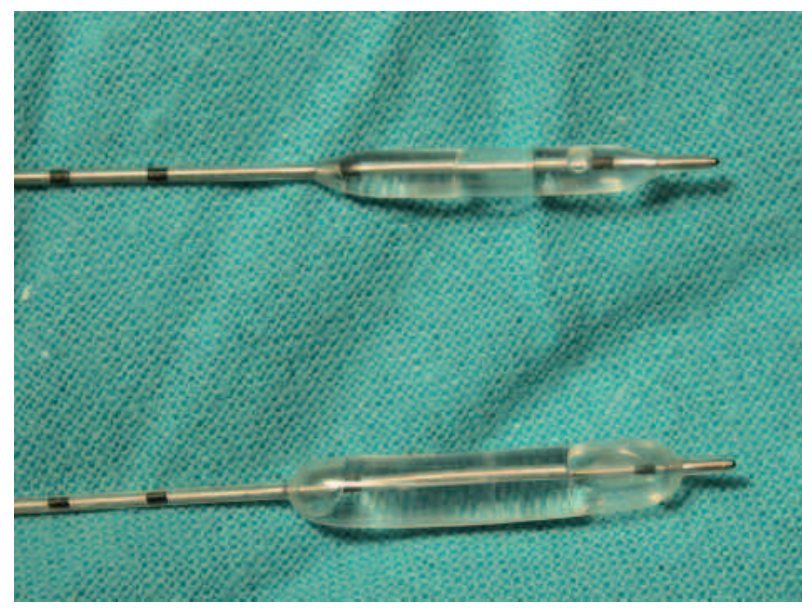

Fig. 2: Inflado de balón de $2 \mathrm{~mm}$. (superior) y de $3 \mathrm{~mm}$. (inferior).

El balón se inflaba durante 90 segundos a una presión de 8 atmósferas, seguidamente se desinflaba y se volvía a inflar a la misma presión durante 60 segundos, acto seguido se desinflaba, lo que permitía su desplazamiento, y colocándolo proximalmente a nivel de la unión de saco con el conducto nasolagrimal coincidiendo el punto lagrimal con la marca distal del catéter queda el punto a 5 milímetros de la porción proximal del balón y se repetía la misma secuencia de inflado de 90 segundos, desinflado e inflado de 60 segundos a la misma presión, tras desinflarlo se extraía el balón del sistema lagrimal y se lavaba con suero salino con fluoresceína. El fluido normalmente se irriga fácilmente después de la dilatación no debiendo encontrarse ninguna resistencia, se confirma la permeabilidad mediante control endoscópico. Se aspiraba el fluido con un tubo del numero 8 , además con esta maniobra se limpiaba la vía lagrimal excretora.

El tiempo medio de la cirugía fue de 7 minutos (rango 6-10).

Tras la cirugía se prescribía un colirio antibióticocorticoideo cuatro veces al día 10 días y gotas nasales antibiótico, corticoideas y vasoconstrictoras pediátricas con Oximetazolina al $0,025 \%$ tres veces al día durante cinco días. Se prescribió tratamiento sistémico excepto si encontraba abundante secreción purulenta al lavado de la vía lagrimal; en este caso Cefuroxima $15 \mathrm{mg} / \mathrm{kg} /$ día se prescribió durante 1 semana.

Se evaluó al paciente a las dos semanas, tres meses y seguidamente cada 6 meses.

En ningún paciente se repitió el proceso. 
El seguimiento fue entre 6 y 21 meses (media 15 meses).

En cada visita se practicó el test de desaparición de colorante con una gota de fluoresceína sódica al $2 \%$ en cada fondo de saco conjuntival inferior estudiando a los 5 minutos la tinción residual (tabla II), se preguntó al tutor por la presencia de secreción y epífora cuantificando ésta con test de Munk, y se examina si existía regurgitación o distensión a la presión sobre el saco.

Se clasificaron los resultados como buenos si desaparece el lagrimeo y la secreción mucopurulenta y el colorante desaparecía del menisco lagrimal (Munk 0 y tinción grado 0), aceptables si el lagrimeo mejoraba en más de dos grados en la escala de Munk pero no desaparecía totalmente y el colorante a los 5 minutos persistía (grado 1 y 2), y malo si el resultado era igual a como estaba el paciente previamente, mejoría menor o igual a 1 grado en la escala de Munk y retención del colorante en grado 3 ó 4.

El test estadístico aplicado fue el test $\chi^{2}$ con corrección de Yates con un nivel de confianza del $95 \%$.

\section{RESULTADOS}

Técnicamente se realizó la cirugía en el 100\% de los pacientes.

Todos los pacientes fueron estudiados por más de 6 meses después de la cirugía (rango 6 a 21 meses).

El $50 \%$ de los pacientes tuvieron obstrucción membranosa a nivel de la válvula de Hasner durante el pasaje de la sonda. En estos pacientes el resultado fue bueno en un $80 \%$ de los pacientes, aceptables en un $13,33 \%$ de los pacientes y pobre en un $6,66 \%$. Un paciente presentó regresión de los resultados siendo bueno (Munk 0 y test de tinción 0) en la revisión de las dos semanas y aceptable (Munk 2 y test de tinción 1) en la revisión de los tres meses.

Ocho pacientes tuvieron obstrucción completa lagrimal, el resultado fue bueno en un $75 \%$ de los pacientes y malo en el $25 \%$.

\section{Tabla II. Test de desaparición de colorante}

Grado 0 Sin tinción residual a los 5 minutos.

Grado 1 Tinción a los 5 minutos menor o igual al 25\% inicial. Grado 2 Tinción a los 5 minutos entre el $26 \%$ y $50 \%$ inicial. Grado 3 Tinción a los 5 minutos entre el $51 \%$ y $75 \%$ inicial. Grado 4 Tinción a los 5 minutos entre el $76 \%$ y $100 \%$ inicial
En los siete pacientes con obstrucción parcial encontramos resultados buenos en el 100\% (fig. 3).

Las observaciones más relevantes de este trabajo estriban en que el resultado mayoritario independientemente del tipo de obstrucción fue el considerado como «bueno»: en la obstrucción tipo estenosis fue del 100\%; para la proximal del $75 \%$ (EE: $\left.30 \% \mathrm{IC}_{95 \%}: 45 \%-100 \%\right)$ y para la membranosa del 80\% (EE: 20,24\% $\mathrm{IC}_{95 \%}$ : 59,76\%-100\%).

En este estudio se obtiene una $X_{\text {exp }}^{2}=1,005 \mathrm{y}$ $X^{2}{ }_{4,0.95}=9,4877$, como $X^{2}{ }_{\exp }<X_{4,0.95}^{2}$, se deduce que no existe dependencia estadística entre las variables cualitativas tipo de obstrucción y resultados. Por tanto el tipo de obstrucción no influye en los resultados obtenidos.

En cuanto a la distribución por edad el grupo 1 (pacientes de hasta 36 meses de edad) fueron incluidos 16 pacientes.

Los resultados en el grupo 1 fueron buenos en un $81,25 \%$ de los pacientes, aceptables en un $6,25 \%$ y malos en un $12,5 \%$.

En el grupo 2 (mayores de 36 meses) fueron incluidos 14 pacientes.

Los resultados en el grupo 2 fueron buenos en un $85,71 \%$ de los casos, aceptables en un $7,14 \%$ y malos en un $7,14 \%$ (fig. 4 ).
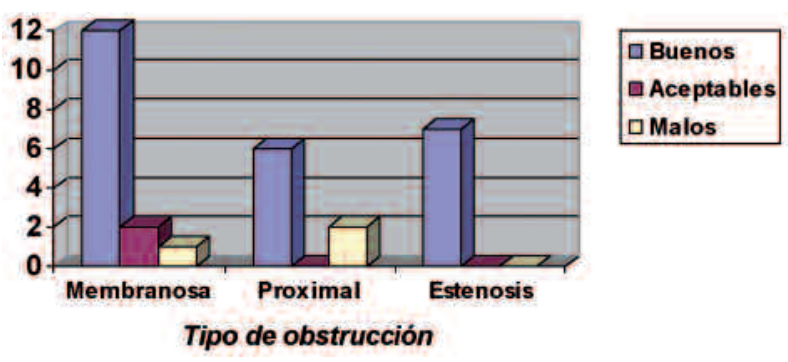

Fig. 3: Relación entre el tipo de obstrucción y resultados obtenidos.

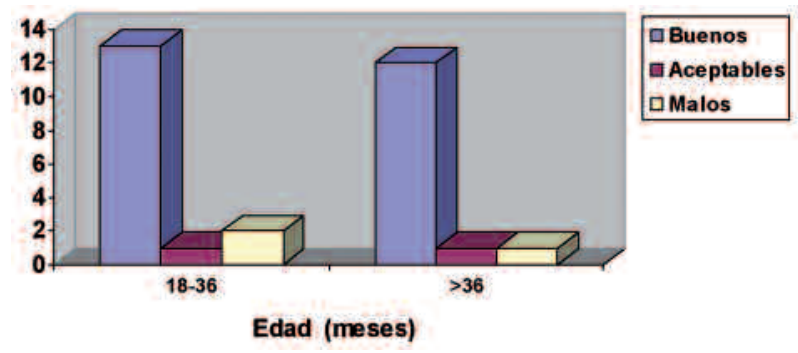

Fig. 4: Relación entre la edad del paciente y resultados obtenidos. 
Las observaciones más relevantes de este trabajo estriban en que el resultado mayoritario independientemente de la edad de los pacientes fue el considerado como «bueno»: en el grupo 1 fue del $81,25 \%$ (EE: $19,12 \%$ con $\mathrm{IC}_{95 \%}: 62,13 \%-100 \%$ ) y para el grupo 2 del $85,71 \%$ (EE: $18,33 \%$ con $\mathrm{IC}_{95 \%}$ : $67,38 \%-100 \%)$.

En este otro estudio se obtiene una $X^{2}{ }_{\exp }=0,3947$. Este valor lo tenemos que comparar $\operatorname{con} X_{2,0.95}^{2}=5,9915$. Al ser $X^{2}{ }_{\exp }<X^{2}{ }_{2,0.95}$, no existen evidencias para rechazar la hipótesis de independencia a un nivel de confianza del 95\%. La edad tampoco influyó en nuestros resultados.

\section{DISCUSIÓN}

La obstrucción lagrimal congénita afecta aproximadamente entre un $6 \%$ y un $20 \%$ de los recién nacidos. La curación es espontánea en la mayoría de los casos en el primer año. En los casos en los que no se produce resolución al año, un primer sondaje es efectivo en un $90 \%$ de los casos, curando además con un segundo sondaje un $6 \%$ más de los pacientes. Hay un grupo de pacientes que no responden al sondaje. Factores de riesgo de fracaso son la edad del paciente, sondaje previo fallido, falsas vías, apertura demasiado pequeña, obstrucción bilateral, saco lagrimal dilatado y obstrucciones completas no membranosas (3-5).

En los casos donde encontramos resistencia al sondaje podríamos indicar la DCP de entrada, además de los casos de sondajes fallidos, está indicación se podría ampliar también como cirugía de primera elección en mayores de dos años $(3,6,7)$.

Estamos de acuerdo con las series de Chen y con los comentarios de Kushner en la que la DCP probablemente no esté indicada en todos los niños, casos con obstrucción simple pueden responder al sondaje sólo.

En casos de obstrucción parcial Yüksel encuentra un $100 \%$ de éxitos en los cuatro pacientes que realiza (6). En nuestros casos se produjo curación en los siete pacientes con vía permeable y epífora.

El sondaje actúa solamente de forma puntiforme a nivel de la obstrucción. Una importante ventaja de la DCP con respecto al sondaje o a la intubación es la aplicación de una fuerza eficiente a lo largo del ducto nasolagrimal de forma radial y longitudinal de forma directa lo que lleva a una dilatación mayor.

Se realizó la inserción anterógrada del balón por ser una vía a la que esta familiarizado el oftalmólo- go ya que es muy similar al sondaje, lo practicamos con control endoscópico por permitirnos una visualización directa que evita trayectos submucosos y la exposición a radiaciones ionizantes como en el caso de inserción retrograda con fluoroscopia (9).

Becker utiliza antibióticos sistémicos previos para eliminar la infección y friabilidad antes de la cirugía y hacer más efectiva la dilatación. Asimismo emplean corticoides en la cirugía y después de ésta para disminuir el edema post-dilatación y evitar fibrosis secundaria así como para permitir una recuperación más rápida (2). Un estudio controlado del balón con y sin medicación sistémica sería deseable. En nuestra serie no se empleó tratamiento sistémico de forma protocolizada.

Encontramos, como Casas y Prat (1), que la mejoría del paciente se produce generalmente a la semana de la intervención quizá por la inflamación post-quirúrgica inducida.

Es muy interesante que los resultados la dilatación con balón son mucho mejores en casos de obstrucción congénita que en adultos aunque se trate de casos de obstrucción incompleta. En adultos la regresión es la norma como ya comunicamos en el XV Congreso de la Sociedad Española de Cirugía Plástica Ocular y Orbitaria en 2005, Chiclana, Cádiz. Esta diferencia puede ser explicada por diferencias en la patogénesis. En los adultos la epífora suele estar causada por etiología de causa adquirida como estenosis involutiva, inflamación e infección crónica siendo por tanto la incidencia de estenosis secundaria alta debido adhesiones y fibrosis de estos tejidos al contrario que en los casos congénitos (9).

Kushner encontró recurrencias en dos de 23 casos cuando comparó los pacientes entre 6 semanas y 2 meses (4). Chen no encuentra cambios (7).

Encontramos en nuestra serie recurrencias en un paciente.

Chen encuentra peores resultados en niños con obstrucción bilateral congénita, los factores posibles pueden ser: reutilizar el mismo balón, la edad más avanzada de estos casos o quizá un sesgo por el pequeño tamaño de la muestra (7).

Otro tratamiento para los pacientes con CNLDO es la intubación con silicona que actúa como un implante temporal. Es un tratamiento efectivo que precisa de la permanencia de los tubos de silicona en el ducto, si bien estos pueden ir asociados a complicaciones hasta en un $20 \%$ de los casos como son: rotura, pérdida de la sonda y fracaso quirúrgico (11), erosión y ulceración corneal, conjuntivitis de 
repetición, laceración del punto lagrimal, granulomas, estenosis de la vía lagrimal excretora (12) y dacriocistitis. Además la inserción y recogida del tubo puede ser difícil en los casos con hipertrofia del cornete inferior y abundante contenido mucoso con posibles laceraciones y sinequias iatrogénicas (13). La intubación bicanalicular en niños puede requerir además una segunda anestesia general para la retirada de los tubos, no remover los tubos puede ir unida a complicaciones como la obstrucción por granulomas y rinolitos en cavidad nasal.

En nuestra serie no encontramos efectos secundarios con la DCP. Se ha descrito dificultad técnica en su inserción, laceración de puntos lagrimales y canalículos.

Goldstein no observó cambios histopatológicos tras su utilización y análisis en modelos animales (14).

La DCP es un tratamiento sencillo de realizar, requiere menos manipulación nasal pues no hay que introducir pinzas o ganchos para recoger los tubos, reduce por tanto el tiempo quirúrgico, evitando laceraciones de mucosa y posibles sinequias, es bien tolerado, no está asociado a las complicaciones postoperatorias de la intubación bicanalicular y no requiere un segundo paso por quirófano para su retirada disminuyendo por tanto los costes $(15,16)$.

Representa nuestro tratamiento de elección en los casos de fallo del sondaje antes que la intubación y la dacriocistorrinostomía.

\section{BIBLIOGRAFÍA}

1. Casas E, Prat J. Obstrucción congénita conducto lagrimonasal: actitud diagnóstica y terapéutica. Annals d'Oftalmología 2004; 12: 22-29.

2. Becker BB, Berry FD. Balloon catheter dilatation in pediatric patients. Ophthalmic Surg 1991; 22: 750-752.

3. Tao S, Meyer DR, Simon JW, Zobal-Ratner J. Success of balloon catheter dilatation as a primary or secondary pro- cedure for congenital nasolacrimal duct obstruction. Ophthalmology 2002; 109: 2108-2111.

4. Kushner BJ. Balloon catheter dilatation for treatment of congenital nasolacrimal duct obstruction. Am J Ophthalmol 1996; 122: 598-599.

5. Paul TO, Shepherd R. Congenital nasolacrimal duct obstruction: natural history and the timing of optimal intervention. J Pediatr Ophthalmol Strabismus 1994; 31: 362367.

6. Yuksel D, Ceylan K, Erden O, Kilic R, Duman S. Balloon dilatation for treatment of congenital nasolacrimal duct obstruction. Eur J Ophthalmol 2005; 15: 179-185.

7. Chen PL, Hsiao CH. Balloon dacryocystoplasty as the primary treatment in older children with congenital nasolacrimal duct obstruction. J AAPOS 2005; 9: 546-549.

8. Clark RA. Dilation probing as primary treatment for congenital nasolacrimal duct obstruction. J AAPOS 2002; 6 : 364-367.

9. Cho YS, Song HY, Ko GY, Yoon CH, Ahn HS, Yoon HK. Congenital lacrimal system obstruction: treatment with balloon dilation. J Vasc Interv Radiol 2000; 11: 13191324.

10. Gunton KB, Chung CW, Schnall BM, Prieto D, Wexler A, Koller HP. Comparison of balloon dacryocystoplasty to probing as the primary treatment of congenital nasolacrimal duct obstruction. J AAPOS 2001; 5: 139-142.

11. DeRespinis P, Coats D, Gold R. Management of nasolagrimal duct obstruction: ballons, tubes and timing. $J$ Pediatr Ophthalmol Strabismus 2006; 43: 73-76.

12. Alañón F, Alañón M, Martínez A, Cárdenas M, Junceda J. Intubación lagrimal en casos complicados. Arch Soc Esp Oftalmol 2006; 81: 313-314.

13. Becker BB, Berry FD, Koller H. Balloon catheter dilatation for treatment of congenital nasolacrimal duct obstruction. Am J Ophthalmol 1996; 121: 304-309.

14. Goldstein SM, Katowiz JA, Syed NA. The histopathologic effects of balloon dacryoplasty on the rabbit nasolacrimal duct. J AAPOS 2006; 10: 333-335.

15. Goldstein SM, Goldstein JB, Katowitz. JA. Comparison of monocanalicular stenting and balloon dacryoplasty in secondary treatment of congenital nasolacrimal duct obstruction after failed primary probing. Ophthal Plast Reconstr Surg 2004; 20: 352-357.

16. Casady DR, Meyer DR, Simon JW, Stasior GO, ZobalRatner JL. Stepwise treatment paradigm for congenital nasolacrimal duct obstruction. Ophthal Plast Reconstr Surg 2006; 22: 243-247. 